\title{
MODEL OF HEAT SIMULATOR FOR DATA CENTERS
}

\author{
JAN NOVOTnÝ*, JIŘÍ NoŽIČKA \\ Department of Fluid Dynamics and Thermodynamics, Faculty of Mechanical Engineering, CTU in Prague, \\ Technická 4, Prague 6, 16607, Czech Republic \\ * corresponding author: Jan. Novotny@fs. cvut.cz
}

\begin{abstract}
The aim of this paper is to present a design and a development of a heat simulator, which will be used for a flow research in data centers. The designed heat simulator is based on an ideological basis of four-processor $1 \mathrm{U}$ Supermicro server. The designed heat simulator enables to control the flow and heat output within the range of $10-100 \%$. The paper covers also the results of testing measurements of mass flow rates and heat flow rates in the simulator. The flow field at the outlet of the server was measured by the stereo PIV method. The heat flow rate was determined, based on measuring the temperature field at the inlet and outlet of the simulator and known mass flow rate.
\end{abstract}

KEYwORDS: data center; cooling; heat simulator; PIV; measurement.

\section{INTRODUCTION}

With the increasing performance of the data centers [1], the requirements for the installed cooling capacity and uniform distribution of the cooled air inside the data center (DC) are growing. The data center management, its users and providers impose very high requirements on perfect operation of DC. DC providers declare failures in availability of the data saved on data center servers in order of a few minutes during one year. Therefore, it is not possible to design DC and implement additional modifications, which optimize the cooled air distribution, during the data center operation. The overheating, destruction of servers and subsequently the loss of data saved on servers caused by inappropriate design of DC is completely unacceptable. In order to ensure optimal air distribution to individual servers, it is necessary to perform, before designing the DC, a numerical simulation, which would help to choose an appropriate layout of DC [2 5]. The numerical analysis must be based on verified numerical model, including appropriately chosen simulation of boundary conditions, sufficient quality mesh and adequate numerical solver [6, 7]. In order to verify that the chosen numerical solution can be used for simulation of flow field and temperature field inside the data center, the model must first be verified with the help of real experiments. Since we could not, during our work, perform adequate measurements inside the real data center and thus correct the numerical simulations based on those measurements, it was necessary to implement the model case of the data center with at least one data rack and to verify the numerical solution in that manner. In order to implement such solution, the design and verification of a suitable heat simulator, which would take over the role of servers, was necessary. This work follows the results presented at the conference EFM2014 [8].

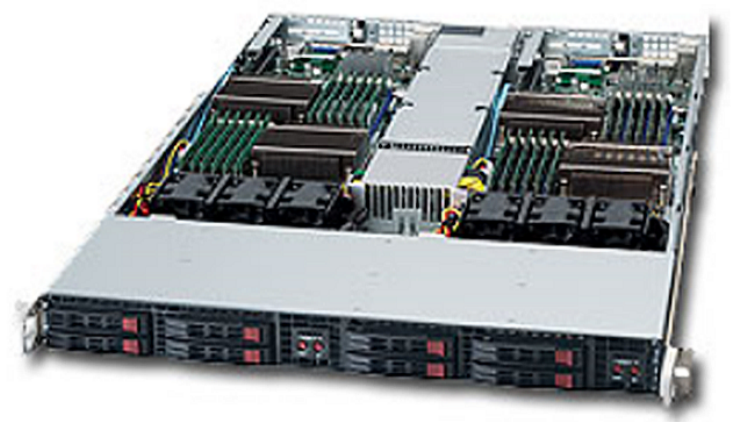

Figure 1. Server Supermicro 1026TT. From http://www. supermicro.com/products/system/1U/ 1026/SYS-1026TT-TF.cfm [2016-08-01].

\section{Heat Simulator OF SERVER}

Before designing the heat simulator, we had to choose a suitable template for our model. The Supermicro 1026TT-IBQF1200W server (Figure 1) was chosen as a suitable ideological basis. This server can be fitted with up to four processors, each with an output of $130 \mathrm{~W}$; and each processor has six slots for memory modules. Depending on the manufacturer, the heat output of each memory module is about 20W [6, 9. A group of six fans with a suction and delivery part is located in the central part of the server. The battery is located inside the server. In the front part, it is possible to connect hard drives or other adequate equipment. With a view to ensuring the best approximation of our heat simulator, we have decided to keep all the major parameters consistent with Supermicro 1026TT server. The height of our simulator is identical - $1 \mathrm{U}$, the processors are replaced by heating plates with resistance spirals with an output of $150 \mathrm{~W}$. Powerful Thermaltake CL-P263 chillers are used for dissipation of heat from the heating plates. A group of four memory modules is simulated by a heating plate with an output of $100 \mathrm{~W}$, and the memory modules 


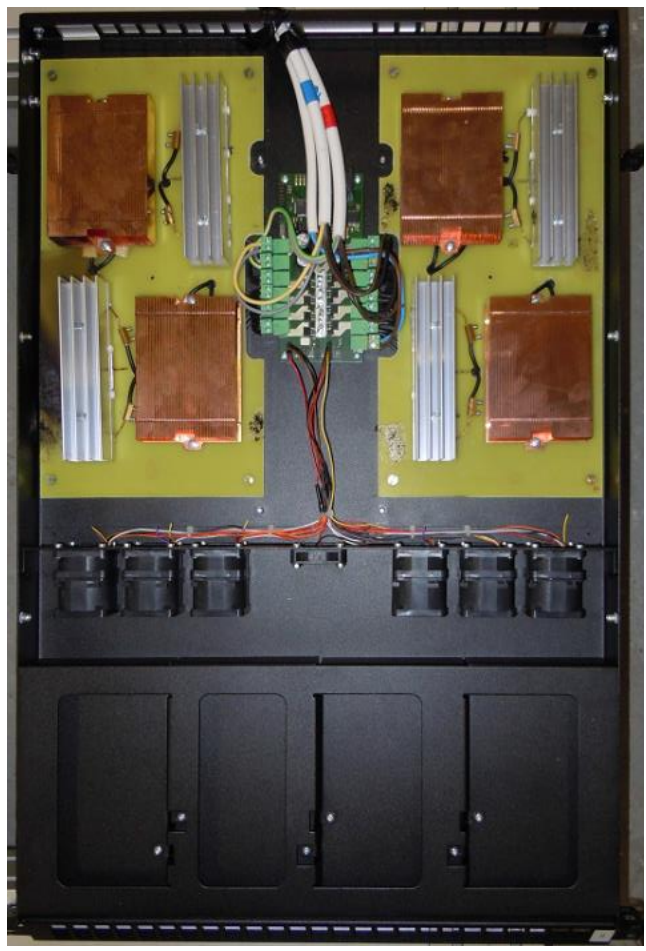

Figure 2. Designed and implemented heat simulator.

are replaced by an ribbed aluminum profile. In the middle, there is a control unit that ensures continuous regulation of the output within the range of 10-100\%. Powerful San Ace 40 fans are located in the central part; four hard drives are simulated at the inlet. Total installed heat output is $1000 \mathrm{~W}$. View of exposed heat simulator is shown in Figure 2

\section{MeAsurement of PERFormance CHARACTERISTIC OF HEAT SIMULATOR}

For the purpose of verifying the functionality of the designed model, it was necessary to carry out several basic measurements. One of the major parameters is the mass flow rate in the heat simulator, distribution of velocities and temperature at the outlet and the temperatures of individual simulated components, such as processor and memory module. The measurement of the mass flow rates and distribution of velocities at the outlet of the server were implemented with the help of the stereo PIV method [10, 11]. The temperature field at the outlet of the servers was measured by a set of thermocouples. Temperatures of individual simulated components were controlled by thermocouples again. Our model is very narrow, since it is a model of $1 \mathrm{U}$ server, thus the outlet velocity profile is rectangular in shape. When taking measurements using the stereo PIV method, we proceeded from the requirement of mapping the velocity field at the outlet of the server. For these reasons, the whole flow field had to be divided into five parts in those subsectors and it was necessary to measure the mean velocity field and to compute the total velocity field by

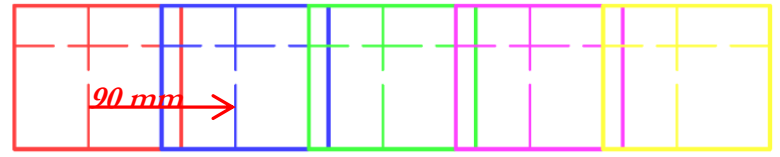

FiguRE 3. Division of the velocity field at the outlet into five subregions, in which the measurement using the stereo PIV method was subsequently carried out.

integrating these results together. The mutual shift between different areas was $90 \mathrm{~mm}$ and the area of the overlap was a minimum of $10 \%$. The division of the outlet flow field into particular subregions is shown in Figure 3. The measurement of the velocity field was carried out for simulator Individual measured 3D velocity fields are shown in Figure 4

The resultant flow characteristic - determined with the help of the Matlab program - is based on the following equation:

$$
\dot{m}=\sum_{x} \sum_{y} c_{w(x, y)} A_{\mathrm{e}} \varrho_{\mathrm{vz}}
$$

where $c_{w(x, y)}$ is the local velocity of $\mathrm{z}$ component; $A_{\mathrm{e}}$ is the surface of the elementary area; $\varrho_{\mathrm{vz}}$ is the air density.

The resultant mass flow rate in the heat simulator, determined with the help of the stereo PIV method, is shown in Figure 5

A detailed measurement of properties of the designed heat simulator also includes the measurement of temperature fields and temperatures of individual simulated components. The measurement was carried out by a set of T-type thermocouples. During the measurement, we used a NI 9215 card for sixteen thermocouples and a slot for NI cDAQ-9178 cards. For the purpose of data recording and subsequent evaluation, a LabVIEW program was used. The connection of individual thermocouples is shown in Figure 6. The Figure shows a set of sixteen thermocouples (red) used for mapping the temperature field at the outlet from the server. Figure 6 shows the connection of ten thermocouples with the use of which (blue) the temperature conditions inside server and in its immediate vicinity were observed. Only half of the „memories” and temperature CPU at the inlet and outlet was observed. Total temperature field at the outlet was measured with the help of 16 thermocouples in a row and three positions from the top of the server $(10,20$ and $30 \mathrm{~mm}$ from the upper lid of the server). The resultant temperature field was composed of these measured temperatures and was measured in dependence on the performance of fans and heating within the range of 50-100\%. The resultant temperature field is shown in Figure 7] As already mentioned above, the measurement of temperatures of individual simulated components was carried out in order to find out if our designed heat simulator is similar not only as for the maximum heat flux and flow rate but also as for the 
Fan performance 20\%

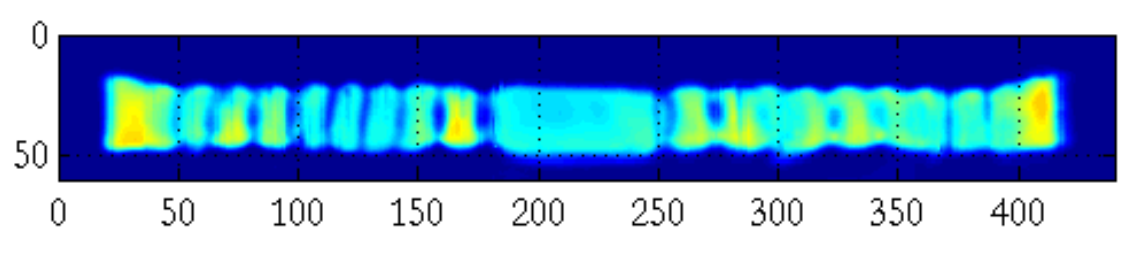

Fan performance $40 \%$

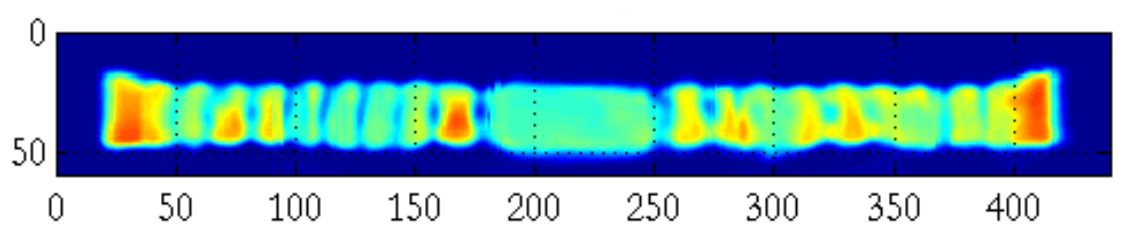

Fan performance $60 \%$

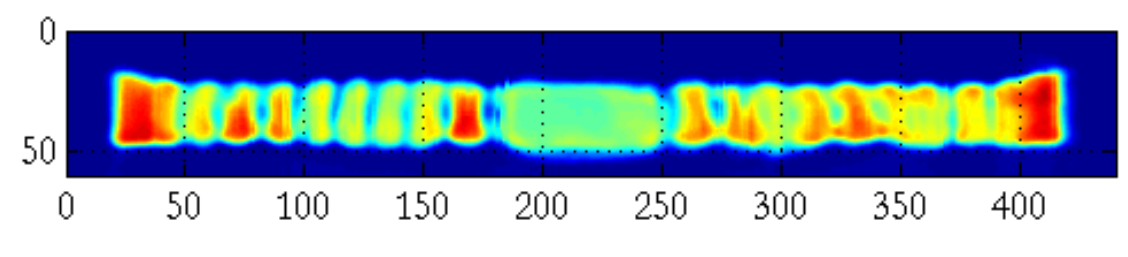

Fan performance $80 \%$

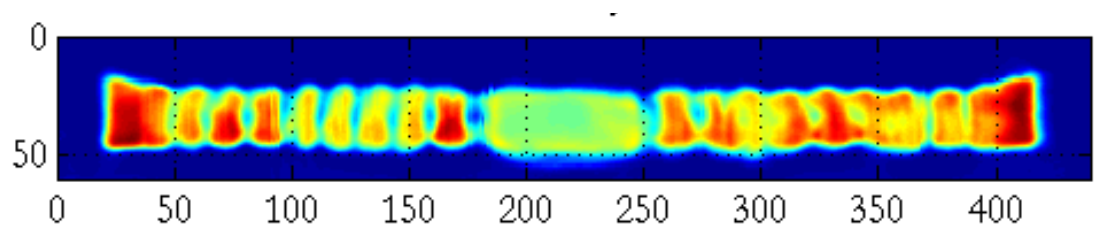

Fan performance $100 \%$

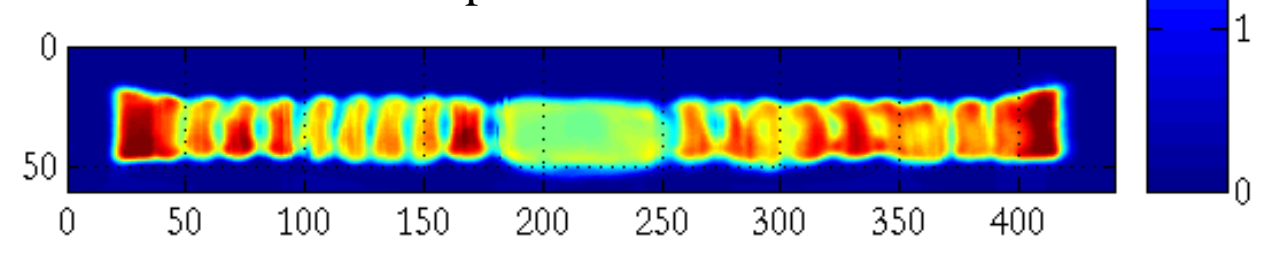

FiguRE 4. Outflow temperature from heat simulator.

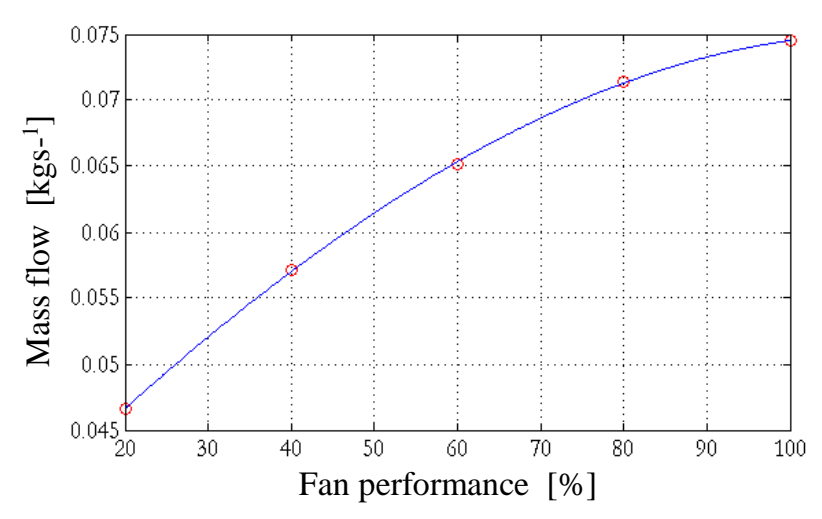

FiguRE 5. Dependence of mass flow rate in server on fans performance.

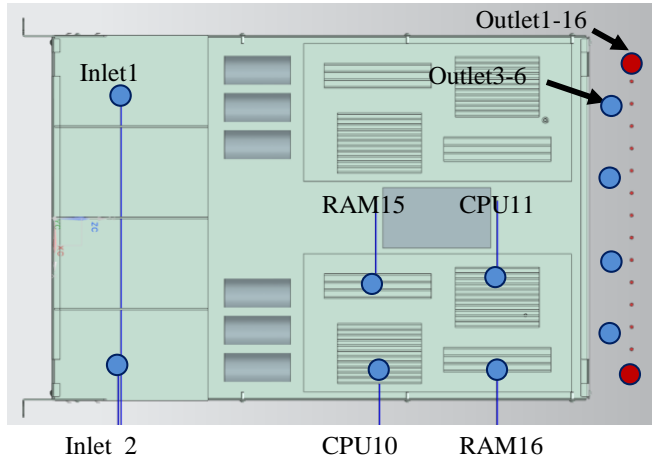

Figure 6. Connection of thermocouples inside the heat simulator. 
Heat performance 50\% Fan performance 60\%
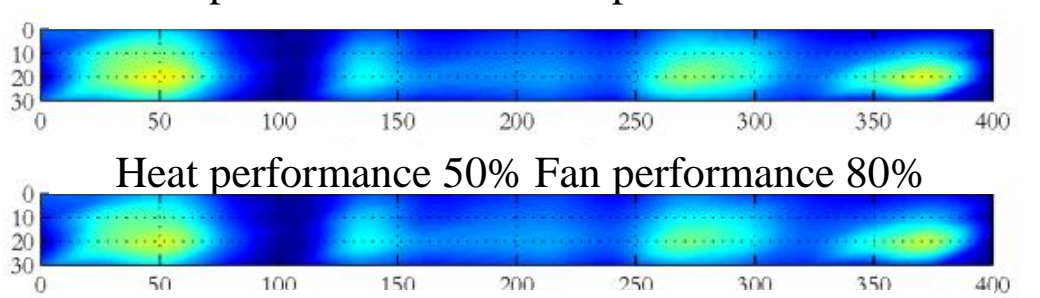

Heat performance $50 \%$ Fan performance $100 \%$

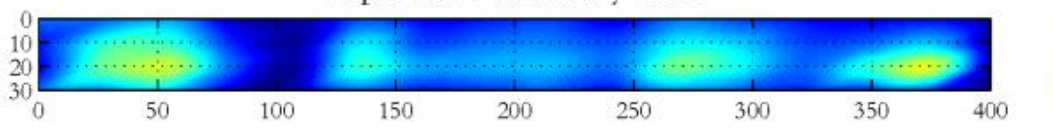

Heat performance $75 \%$ Fan performance $60 \%$

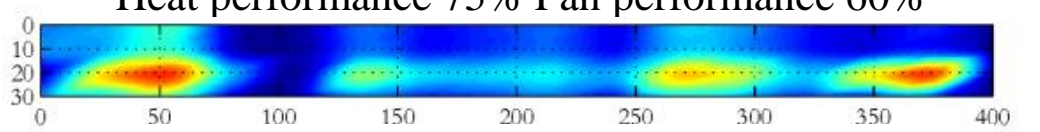

Heat performance $75 \%$ Fan performance $80 \%$

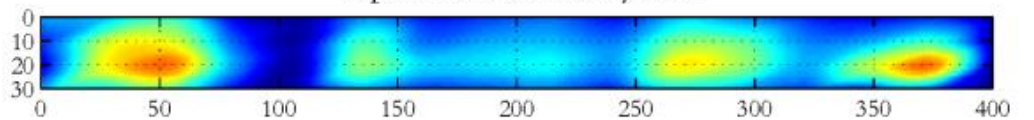

Heat performance $75 \%$ Fan performance $100 \%$
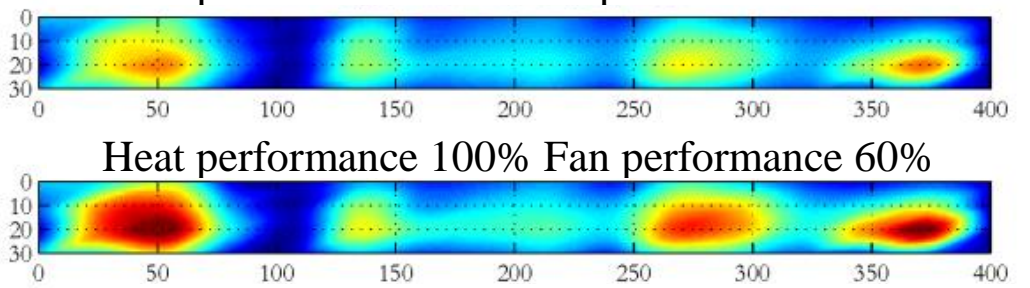

Heat performance $100 \%$ Fan performance $80 \%$

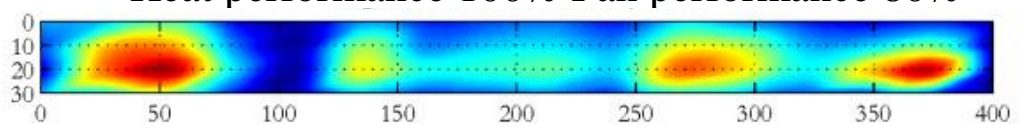

Heat performance $100 \%$ Fan performance $100 \%$

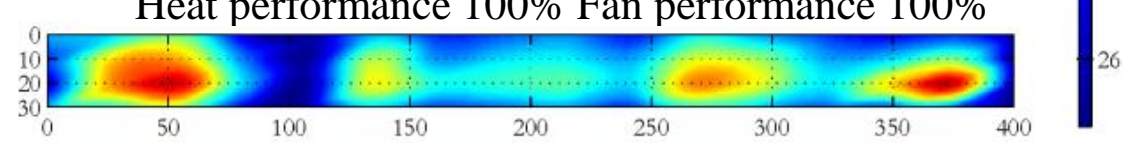

FiguRE 7. Temperature field at the outlet of heat simulator.

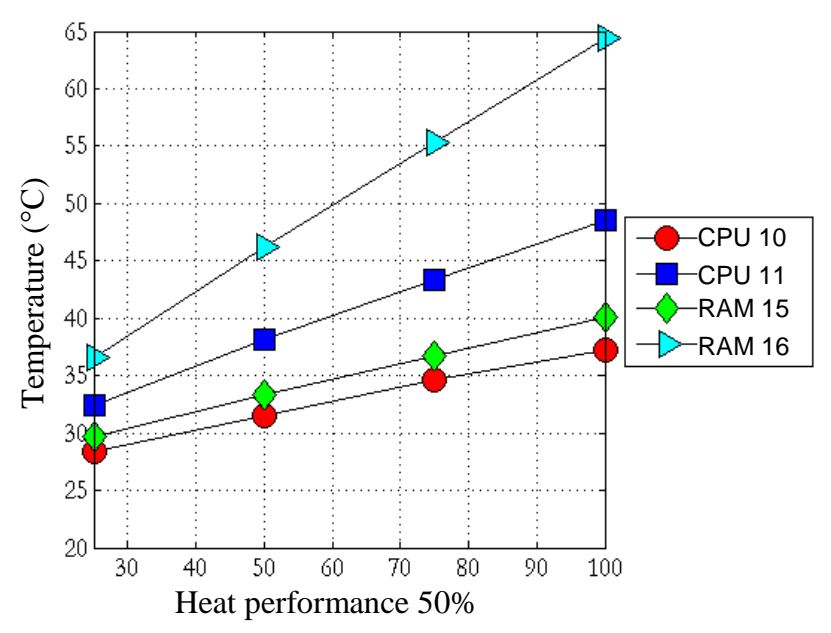

Figure 8. Course of temperatures in dependence on performance of heating units.

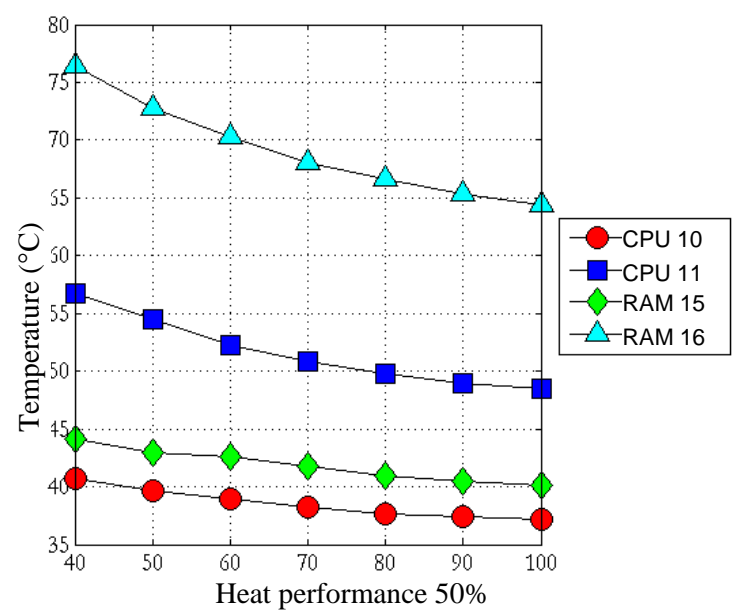

Figure 9. Course of temperatures in dependence on performance of heating units. 
temperatures of individual components and the associated maximum temperatures at the outlet. Our goal is to operate the simulator in a mode that corresponds to the operation of real server in a maximum way. For this reason, the temperature of individual components in dependence on heating power and in dependence on performance of fans was observed (Figures 8 and 9 ). The given dependence clearly shows that, at maximum power of fans, the server can be operated in any server mode and the individual components are not overheated above $70^{\circ} \mathrm{C}$, Figure 8. The situation is different, when keeping the maximum heat flux and reducing the performance of fans. The limit value of $70^{\circ} \mathrm{C}$ is reached in the memory module RAM 16 with fans running at $60 \%$. The temperature of the RAM 16 module increases with further reduction in the performance of fans. Other components in its entirety indicate a significantly lower temperature, which is caused by their closer location to the fans and due to significantly larger heat transfer surface of the CPU11 compared to the RAM 16 module. It clearly follows that if we want the heat simulator to be operated in conditions, which are comparable to the real server operation, it is necessary to set the performance of fans to a minimum of $60 \%$.

\section{Conclusions}

Based on the performed measurement, it may be concluded that the designed and tested heat simulator meets the basic requirements for similarity with the real server. Chosen geometry closely replicates the original Supermicro 1026TT server in terms of the shape and internal layout of basic components. Furthermore, the proper function of heat simulator within a wide range of operation modes was verified. The minimum value of performance of fans and also the corresponding heat flux in the server was determined $0.065 \mathrm{~kg} \mathrm{~s}^{-1}$. Furthermore, the complete velocity field at the outlet of the server was determined using the PIV measurement. Finally, the distribution of the temperatures at the outlet of the server was determined with the help of a set of thermocouples. In the subsequent step, the presented outputs will be used for the purpose of design and verification of suitable numerical model simulating real server fitted in the data rack of the data center.

\section{ACKNOWLEDGEMENTS}

This research has been realized using the support of Technological Agency, Czech Republic, program alpha: Research and development of IT racks, cooling and transport systems for data centers (TA01010184). This research has been supported by Centre for research of multiphase flow and thermodynamics processes in renewable sources and energetics - NEW ENERGY reg. n. CZ.2.16/3.1.00/22130 supported by European Union

\section{REFERENCES}

[1] M. J. Ellsworth, G. F. Goth, R. J. Zoodsma, A. Arvelo, L. A. Campbell and W. J. Anderl, An Overview of the IBM Power 775 Supercomputer Water Cooling System, Journal of Electronic Packaging, vol. 134, 2012 DOI:10.1115/1.4006140

[2] B. D. Estebe, C. Le Bot, J. N. Mancos, E. Arquis, Data Center Optimization Using PID Regulation in CFD Simulations, Energy and Buildings, 66 ,2013, DOI:10.1016/j.enbuild.2013.07.053

[3] D. Moss, J.H. Bean, Energy impact of increased server inlet temperature APC, White Paper, 138, (2011).

[4] A. Vallury and J. Matteson, Data Center Trends Toward Higher Ambient Inlet Temperatures and the Impact on Server Performance, Volume 2, Thermal Management; Data Centers and Energy Efficient Electronic Systems, 2013. DOI:10.1115/IPACK2013-73133

[5] T. Brunschwiler, B. Smith, E. Ruetsche, and B. Michel, Toward zero-emission data centers through direct reuse of thermal energy, IBM J Res Dev, vol. 53, no. 11, pp. 13, 2009.

[6] R.Tessier,V. Betz, D. Neto, T. Gopalsamy, Poweraware RAM Mapping for FPGA Embedded Memory Blocks, Proceedings of the 2006 ACM/SIGDA 14th international symposium on Field programmable gate arrays, 2006. DOI:10.1145/1117201.1117229

[7] B. D. Estebe, C. Le Bot, J. N. Mancos, E. Arquis, Data center optimization using PID regulation in CFD simulations, Energy and Buildings 66, 2013. DOI:10.1016/j.enbuild.2013.07.053

[8] J. Novotný, J. Nožička, J. Jansa, Model of Heat Simulator Data Centers, Proceeding of the EFM 2014, p. $389-393,2014$

[9] J. G. P. Koomey, "Growth in data center electricity use 2005 to 2010," Analytics Press, Oakland, CA, 2011, Analytics Press.

http://www. analyticspress.com/datacenters.html [2016-08-01].

[10] Raffel, M., Willert, C.E., wereley, s., Kompenhans, J. Particle Image Velocimetry A Practical Guide,

Springer-Verlag Berlin Heidelberg. DOI:10.1007/978-3-540-72308-0, 2007

[11] Novotný, J.: Influence Of Data Quality On Piv Measurement Accuracy. Journal of Flow Visualization and Image Processing. 2012, vol. 19, no. 3, p. 215-230. ISSN 1065-3090. DOI:10.1615/JFlowVisImageProc.2013003304 